\title{
DIALECTICA Y LIBERTAD EN CIENCIAS DE LA SALUD
}

Luisa Meyer*

SÍNTESE - Las ciencias médicas manifiestan más crudamente que otras las limitaciones del modelo de ciencias como compartimentos estancos, con métodos de constatación y coherencia lógica estricta. La convocatoria interdisciplinaria requiere de una fundamentación filosófica que, por principio, admita los cambios en el mismo cuerpo conceptual. Se propone aquí una concepción dialéctica porque ella: 1. permite abordar problemas que, debido a sus múltiples y contrarios aspectos, son captables sólo mediante el método de planteos y formulaciones conscientemente cambiantes; 2 . establece, por principio, el carácter limitado y contingente de toda verdad científica; 3 . justifica la convocatoria de comunicación y cooperación de las disciplinas entre sí; 4. El momento de la negación constituye tanto la reflexión autocrítica de la ciencia sobre sus propios límites como su estructura de organismo viviente $\mathrm{y}$ abre el camino hacia verdades nuevas; 5 . en ciencias de la salud el sujeto paciente encarna el momento de la negación; 6. el cáracter inacabado de la verdad científica deja abierta la cuestión de los resultados últimos y permite la libertad de la ciencia y del sujeto que la construye, de la cual es su principio.

PALAVRAS-CHAVE - Dialéctica. Ciencias médicas. Libertad.
ABSTRACT - The different medical sciences show, at times quite clearly, the limitations of a model based on isolated compartments using methods of verification and strict logical procedure. The call to an interdisciplinary approach requires a philosophical foundation which, on principle, admits the changes in the very structure of the concept. This article proposes a dialectic conception because: 1 . it allows a tackling of problems which, because of the diversity, multiplicity and controversity of their components, can only be grasped by a method that permits and enables consciously changing statements and formulations; 2 . it establishes, on principle, the limited and contingent character of any scientific truth; 3 . it justifies the call for communication and cooperation between the various branches of science; 4 . the moment of negation constitutes both the self-critical reflection, on the part of science, on its own limits as well as on its structure as a living organism, opening the way towards new truths; 5 . in medical sciences the patient-subject embodies the moment of negation; 6 . the unfinished character of the scientific truth leaves the question of the final results open and permits the freedom of science and of the subject who is the principle of science. KEY WORDS - Medical sciences. Dialectics. Freedom.

El título del presente trabajo puede parecer, a primera vista, un poco caprichoso en un encuentro sobre dialéctica; no obstante creo que el mismo logra su justificación en tanto puede fundamentar la convocatoria de comunicación y

* Professora da Universidade de Buenos Aires.

\begin{tabular}{|l|l|l|l|l|l|} 
VERITAS & Porto Alegre & v. 44 & n. 4 & Dezembro 1999 & p. 1011-1018 \\
\hline
\end{tabular}


cooperación de las disciplinas entre sí, en este caso de las ciencias de la salud, como también el crecimiento científico y la libertad de la ciencia. Una posición científica que asume el paradigma de su crecimiento debería poder justificar desde sí misma su disposición de apertura a campos de verdad nuevos $y$, consecuentemente también, hacia otras perspectivas metódicas. Pero es a la filosofia a quien atañe la cuestión tanto del objeto como del método de investigación en cientia.

En realidad lo que entra aquí en juego y constituye el verdadero meollo de la cuestión es el concepto de ciencia y su fundamentación. Bien sabemos que todo concepto de ciencia tiene como soporte una comprensión de mundo y de la experiencia del mismo que excede el marco conceptual de aquélla y que, en verdad, atañe a la reflexión filosófica. A ella conesponde ampliar el horizonte, limitado por principio, de toda investigación científica, debido al tipo de razonamiento. específico de ésta. El conocimiento científico específico exige la limitación a un determinado aspecto de la cuestión-objeto y, concordantemente, esta limitación se mantiene a lo largo de todo el curso del razonamiento. Y es a este principio delimitante que las investigaciones científicas deben sus logros.

Pero la concepción de las ciencias como estancos separados, fijos e incomunicados ha puesto en evidencia, desde hace mucho tiempo; su incapacidad tanto para explicar ciertos fenómenos como para resolver determinados problemas. Esto se manifiesta más crudamente que en otros campos en las ciencias médicas. Y los miembros de la comunidad científica de cada campo específico requirieron del auxilio de los otros para abordar tanto la interpretación de ciertos fenómenos como la solución de determinados problemas que, aisladamente, no lograban hacerlo. La convocatoria de otras discipilinas se hizo ineludible.

En el presente trabajo procuro tematizar estas cuestiones abordándolas desde una posición filosófica basada fundamentalmente en la dialéctica hegeliana. Primero analizo el tema de por qué la dialéctica puede dar cuenta del crecimiento científico desde un principio de razón intemo, propio, de la ciencia misma (Dialéctica y crecimiento científico); en segundo lugar trataré el tema del fundamento o del principio de la ciencia en la dialéctica ( $L a$ cuestión del principio de la ciencia: el sujeto) y, finalmente, explicitaré la razón de por qué en esta línea de pensamiento de orientación dialéctica la libertad de la ciencia y de su sujeto no sólo no queda anulada sino por el contrario, es proclamada por principio (Dialéctica y libertad).

\section{Dialéctica y crecimiento científico}

Este reconocimiento de los límites de validez de cada verdad que se establece en la ciencia sólo puede fundamentarse, a mi juicio, en una concepción dialéctica de ésta. Pues en ella se reconoce, por principio, el carácter inacabado de toda verdad científica. Ninguna verdad es $L a$ Verdad, tampoco lo es la última verdad. Toda verdad tiene un campo limitado de validez legítima. Las consecuencias epistemológicas derivables del reconocimiento de esta limitación de principio de la verdad alcanzada demarca el método que puede responder a estas cuestiones: aquél que reconoce, por principio, el carácter limitado de toda verdad científica y por lo tanto su dimensión de contingencia y, en segundo lugar, reconoce en esta dimensión de contingencia el motor generador del crecimiento científico. Se trata de una posición que podemos denominar de escepticismo crítico, en tanto declara 
a priori que toda afirmación verdadera de las ciencias tiene sus propios límites de validez y acepta que ninguna realidad es totalmente abordable en ningún momento del desariollo del crecimiento científico, ni tampoco desde una sola perspectiva de consideración y por eso convoca a las otras disciplinas en el momento en que deviene consciente de sus propios límites. Se sienta asi, por principio, posibilidades de apertura hacia nuevas dimensiones de verdad en la comunicación e integración con otras disciplinas afines, con la filosofía y con otras perspectivas que no necesariamente son de carácter epistemológico - como el arte y la religión.

En el ámbito filosófico en que se reflexiona sobre esta toma de conciencia de los propios límites por parte de las ciencias se pone de manifiesto la necesidad de que las afirmaciones científicas no sean tomadas como hechos dados, sino que deben ser acreditadas o legitimadas, siempre, cada vez nuevamente, en la perspectiva de su objeto de conocimiento. Y esta cuestión sólo puede tener cabida en una posición filosófica que no puede menos que ser dialéctica, entendiendo a ésta no como un método con pretensiones de aplicación directa en una determinada esfera del campo del saber científico sino como meta-reflexión filosófica sobre dicho saber que logra articular una figura explicativa del progreso del mismo desde un principio que no es externo a la ciencia sino propio, endógeno a la mismo. En esta consideración dialéctica sobre las ciencias - en el caso que nos ocupa, de las ciencias médicas - éstas se autodescubren en una configuración que puede dar cuenta de su progreso desde un concepto de verdad que acepta los cambios y puede legitimar su libre ejercicio en tanto investigación.

En el caso de la posición dialéctica a la cual se adhiere aquí - la hegeliana el principio está dado por el mismo proceso de particularización del concepto, que va concretizándose al mismo tiempo que se va ampliando o va incorporando nuevas dimensiones. Pues el principio dinámico forma parte de la misma estructura del concepto.

Subyace aquí el presupuesto de que sólo un método abierto, de conclusión indeterminada, es adecuado para captar la totalidad de la realidad en sus múltiples, diversos y contrarios aspectos. Así, cuando un objeto de estudio resulta imposible de captar o comprender, a pesar de todos los esfuerzos sistemáticos y de lógica coherencia - típicos de métodos de explicación causal lineal - puede que se capte mediante los planteos y formulaciones conscientemente cambiantes de una concepción dialéctica de la ciencia. Lo que este procedimiento procura evitar es la limitación típica de las perspectivas nígidas, fijas.

Esto no significa proponer para la ciencia simplemente reflexiones dispersas, peregrinas y caóticas. Las reflexiones dialécticas tienen que seguir también un principio de orden o metódico. Al respecto afirma, por ej. Cirne-Lima: "En el discurso especulativo cada tesis y cada antitesis son sólo una parte del todo y, como tales, pueden y deben ser fundadas; y deben ser demostradas como falsas a través de razones. Y la refutación es, en verdad, analítica".'

Estas afirmaciones de Cirne-Lima establecen también,a otro nivel, de algún modo, la diferenciación metódica entre ciencia y filosofia. En la dificil tarea de autoreflexión, puesta al día y comunicación permanente ni ciencia ni filosofia

"Razões, fundamentos e causas", Veritas, v. 43, n. 4, p. 808, Porto Alegre, 1998. 
deben confundir sus sendas. La filosofía no debe perder de vista su identidad: debe mantener la impronta de generalidad (el concepto que deviene autoconsciente o para sí es también en la forma de concepto, es decir, de universalidad) y, al mismo tiempo debe hacerse cargo de las determinaciones que va incorporando el concepto en sus campos específicos, o de sus nuevas configuraciones en las que muestra su crecimiento.

Respecto de la dialéctica hegeliana recordemos pues aquí que Hegel denomina, en general, dialéctica al movimiento del espíritu que va estructurando conceptualmente los pensamientos 0 , dicho de otro modo, al movimiento de autodesenvolvimiento de los conceptos ${ }^{2}$ que se da en un proceso cuyos hitos son, como es conocido, tres y suelen mencionarse en la jerga filosófica como tesis, antítesis y síntesis o como afirmación, negación y negación de la negación, fórmula ésta que exige tematizar sus supuestos

En realidad lo que Hegel denomina dialéctica es el método del cual se vale para exponer la realización de todo concepto estructurado por el hombre y presupone un principio energético que desata un proceso de particularización del concepto que, como tal es en sí universal. Este proceso tiene la particularidad de crear desde sí mismo sus propias determinaciones o configuraciones; por eso es preferible hablar de autodesenvolvimiento en vez de desarrollo. Este proceso atraviesa diferentes estadios. Sin embargo, en la transición de un estadio a otro el sujeto permanece siendo el mismo. ${ }^{3}$

Hegel interpreta el autodesenvolvimiento del concepto como un emerger en la realidad externa de la posibilidad existente al comienzo sólo como mera potencia y como curso o devenir en el cual la potencia o posibilidad existente primeramente sólo como simplicidad inmediata se configura en un todo articulado. Se trata de una estructura racional que tiene el "impulso" de salir de su interioridad subjetiva y configurar su "ser en sí" en existencia objetiva. Entiende también este proceso del crecimiento auto-configurativo de los conceptos como un crear un todo articulado comparable a un organismo viviente o principio dinámico, generador de movimiento hacia la forma final articulada.

La dialéctica puede dar razón de la ciencia como organismo viviente: porque concibe a la ciencia como sistema racional conceptual en acto, consciente de sí misma y de sus límites, y, en tanto autoconsciente, en constante devenir. En la dialéctica hegeliana la ciencia tiene la estructura de organismo viviente; es decir, aparece como sistema vivo y articulado racionalmente. La "totalidad" diferenciada u organismo que es la ciencia se halla supuesta cuando Hegel habla de "sistema" y cuando considera "racional" a una "totalidad" tal, justamente porque ella es en sí unidad viva articulada. Por eso Hegel rechaza por principio toda perspectiva unilateral por ser, según él, "abstracta". La unilateralidad del entendimiento, a diferencia de la facultad de la razón, consiste precisamente en entender un todo como mera unidad "abstracta", mientras que la razón, en cambio, tiene la capacidad de comprender, en la unidad de un todo, la multiplicidad-diversidad de sus elementos $\mathrm{y}$, tras la multiplicidad-diversidad de la realidad puede captar la unidad del todo. ${ }^{4}$

Phän. d. G.. Vorr.; WL., I' Ed.Vorr.; Enz, 1830, § 161; Grundlinien d. Ph. d. Rechts., $§ \S 30 / 33$.

WL II, Logik des Begriffs, I, 1,A.

Enz., 1830, § 422 - 439 . 


\section{La cuestión del principlo de la ciencia: el sujeto}

Como acabamos de ver para expresar su concepción Hegel se vale del modelo aristotélico de orientación teleológica: pero por otro lado y fundamentalmente, asume los presupuestos del Idealismo.

El modelo aristotélico: el concepto es principio interno o forma que primeramente es sólo proyecto, posibilidad o potencia; pero, en tanto tal, es principio de movimiento que inicia un proceso que culmina en una forma articulada, acabada precisamente porque ésta estaba ya, desde el comienzo, como fin o causa final del proceso de desenvolvimiento $\mathrm{y}$, por lo tanto, como hilo orientador del mismo. Por eso el concepto, que es forma inmanente en la materia tiene también un carácter dinámico y puede llegar a constituirse en "idea", es decir, puede llegar a efectivizar su forma. $\mathrm{O}$, dicho de otro modo, el concepto ha logrado poner su figura en la realidad de manera efectiva. Los estadios no deben ser interpretados sólo como determinaciones conceptuales, que alguien pudiese efectuar, por ejemplo, en un análisis filosófico lingüístico, sino que son, según Hegel, configuraciones reales. Es decir que el proceso produce algo, genera algo. ${ }^{5}$ Esto último pone en evidencia, como dije, que esta concepción es comprensible no solamente a partir de la teleología natural de Aristóteles. En realidad es necesario tener en cuenta aquí, ante todo, que el presupuesto fundamental es la concepción del espiritu con capacidad de espontaneidad, propia del Idealismo Alemán, es decir, la concepción del espiritu en tanto principio de vida. Aquí el espíritu no sólo tiene capacidad de comprensión sino también y, ante todo, capacidad activa de insertarse en la realidad, estructurándola a su semejanza o dándole su propia figura.

En esta línea de orientación dialéctica se concibe al espíritu como principio estructurador de la realidad $\mathrm{y}$, por lo tanto, como transfiriendo a ésta su estructura. La ciencia, en tanto parte de la realidad, refleja la forma de quien la configura. De este modo, se interpreta al desarrollo científico a partir de una teoría del concepto que es capaz de objetivarse en la realidad llegando a constituir la estructura racional inmanente de la misma. El espíritu que hace ciencia y que, autocolocándose también como objeto de su propia reflexión, encuentra reflejada, en su obra, su propia forma. Lo importante a tener en cuenta aquí es la relación entre espiritu y configuración de la realidad.

En el eje de esta relación el papel protagónico le corresponde al momento de la negación. En la triada dialéctica la negación representa a la infinita capacidad de negatividad de la conciencia que, cuando compara la realidad con el concepto respectivo - estructurado, a su vez, por ella misma - declara y patentiza a lo más interior de sí misma como fundamento último de la tarea especulativa, como el último hontanar de toda actividad, de toda vida de conciencia que configura el mundo. La ciencia que reflexiona sobre sí misma y deviene consciente de sí como organismo vivo, descubre en este acto de reflexión, que el espíritu es principio u origen de todas las ciencias, en tanto las constituye en la medida en que se autocoloca en el objeto del cual se apropia y le otorga su propia figura.

\footnotetext{
${ }^{5}$ Enz., $3^{a}$ Ed., § 213; Grundlinien d. Phi. d. Rechts. § 1, Anm.
} 
El sentido de la exposición que hace Hegel del desenvolvimiento del concepto en estadios según el esquema de la Lógica es presentar el camino a través del cual el espíitu arriba a un adecuado concepto de sí mismo: es decir, se supone que el espiritu es capaz de ponerse a sí mismo como objeto de consideración, con lo cual asume el rol de sujeto y toda su obra llevará su impronta, por eso, la ciencia es, en consecuencia, también sujeto, o lo es en la forma de la subjetividad. ${ }^{8}$ El sujeto se constituye en principio de la ciencia y transfiere a ésta su traza o figura. Su relación con el "objeto" no es una relación con algo meramente extemo. Cuando algo pasa a ser objeto de conciencia ésta le pone, al mismo tiempo, su sello o lo transforma en toda su estructura, cognitiva y práctica - y organiza los datos que ofrece en unidad.

En una posición filosófica que supone, en cambio, a los mútiples conceptos establecidos en las ciencias como autoabasteciéndose por sí solos y tomados aisladamente, sin relación unos con otros, los conceptos carecen de fuerza explicativa y, por lo tanto, de sentido; porque, en verdad, quien los crea, organiza y da vida en una unidad orgánica es el sujeto o, dicho de otro modo, el sujeto es su principio.

Para exponer la importancia que puede tener hoy volver la mirada hacia una filosofia que declara a la subjetividad como principio de la ciencia me resultan muy ilustrativas las siguientes afirmaciones de Dieter Henrich: "Si hoy tiene sentido ocuparse de subjetividad como principio esto supone fundamentaciones que permiten insertar en la situación filosófica problemáticas de nuestro tiempo".?

Y según sea la interpretación de este tema capital del principio de la ciencia será también luego la concepción de la ciencia y del modo de procedimiento respectivo. La representación que el sujeto de la ciencia tiene de sí mismo se constituye en punto de partida o principio de todo el saber científico y traza el camino que éste habrá de recorrer. Pues en este punto se esboza ya una determinada conciencia, o mejor dicho pre-conciencia del tema en cuestión y se bosqueja anticipadamente la dirección del pensar en que finalmente se articulará el planteamiento del problema porque, por lo general, se piensa, concomitantemente, un marco conceptual para las posibles respuestas y esta pre-comprensión respectiva decide, en gran medida, acerca del método según el cual se investigará la cuestión de la cual se trata. Así, el modelo de explicación causal de la ciencia modema responde a una interpretación filosófica analítica del problema del principio. En la línea de orientación dialéctica, en cambio, se trabaja con una forma circular de pensamiento. Conviene recordar aquí lo que afirma Cirne-Lima al respecto: "La posición dialéctica piensa la serie de razones fundantes como una línea circular en la cual el último eslabón se engarza con el primero de tal manera que, en rigor, no se puede decir dónde está el comienzo o el fin. Cada eslabón de la cadena argumentativa es relativo y apunta tanto hacia adelante como hacia atrás. El círculo de razones fundantes-fundadas se constituyen como fundamento último y sólo él es realmente autofundamentado". ${ }^{8}$

Ahora bien, si hablamos de autofundamentación debemos, retomar el tema del sujeto.

Enz.1830, § 377, 381 - 386.

"Subjektivität als Prinzip", Deutsche Zeitscrnift PHILOSOPH, 46, 1, p. 32, Berlin, 1998.

Op. cit., p. 806. 
Se hace necesario aclarar aquí que por sujeto epistemológico activo de las disciplinas que nos ocupan no entendemos solamente a aquel que la ejerce como científico, sino que, en la praxis de las ciencias de la salud también lo es - y no precisamente en último término - el sujeto paciente en tanto encarna el límite o negación de la verdad acabada. Su rol de tal se patentiza cuando desde el ángulo de su posición de objeto-sujeto de las ciencias terapéuticas se enfrenta a la insuficiencia de la verdad de éstas. Los límites de cada verdad establecida en las ciencias médicas son señalados por cada paciente que presenta los síntomas de un malestar cuyo dominio la ciencia no ha logrado aún. En este momento el paciente pareciera constituirse en un sujeto negador de la verdad de la disciplina médica. Pero esta realidad poco satisfactoria y a veces frustrante significa para la ciencia la posibilidad de una toma de conciencia de sus límites y de un despertar en ella del afán de superarlos; sin poder pretender predecir, desde el comienzo, cuál será el punto de arribo.

\section{Dialectica y libertad}

El segundo momento del método dialéctico, el de negación de la verdad establecida, esboza también el camino hacia un punto de arribo del cual sabemos al comienzo solamente que es tal, es decir, fin por alcanzar, pero la cuestión de los resultados permanece como una cuestión abierta, e.d. sienta el carácter impredecible del proceso científico. Es decir, este estadio conlleva insito también, aparentemente de manera paradójica, un momento positivo al constituirse como momento de autoreflexión de la ciencia sobre su propia estructura. Es decir, en la ciencia que reflexiona sobre sí misma, el "concepto en sí" - para emplear la terminología hegeliana - deviene autoconsciente o "para sí" y alcanza una nueva dimensión de verdad. Es el tercer momento.

En el proceso de movimiento dialéctico el momento de la negación es el que sienta o establece la estructura especulativa viva de la autoreflexión de las disciplinas porque es, en verdad, una reflexión crítico-filosófica sobre sus propios caracteres y límites. El concepto científico no convertido en objeto de reflexión especulativa es sólo en sí. No ha convertido éste, su ser en sí, o su contenido racional, en algo autoconsciente y, por lo tanto, no ha sometido a análisis su propia estructura especulativa ni ha devenido consciente de sus límites.

En esta instancia se establece, de manera necesaria, la exigencia de autoexamen permanente de la ciencia y la ineludible convocatoria de comunicación y cooperación con otras disciplinas y perspectivas. Es el momento positivo del devenir autoconsciente.

El movimiento de autoconfiguración del concepto y de su autoobjetivación en la realidad como idea no se da abruptamente, sino que es un proceso gradual en el cual el sujeto del mismo permanece idéntico a sí a pesar de sus cambios. Y al final del movimiento de autodesenvolvimiento - imposible de alcanzar en un tiempo empírico - el espíritu como objeto y el espíritu como su sujeto - e.d. el pensamiento especulativo mismo - son uno, es decir, completamente adecuados uno al otro, de tal modo que el espíritu que se ve a sí mismo en su propia obra, es en este estadio totalmente cabe-sí. 
Si en esta posición filosófica el crecimiento científico logra - a mi juicio - una explicación y una auto-fundamentación plausibles, no es de menor importancia otro tema a tener en cuenta aquí: el de la libertad de la ciencia y del sujeto que la constituye. En esta posición filosófica - la dialéctica - también la libertad de la ciencia queda proclamada por principio. Como acabamos de adelantar, en el momento de la negación la cuestión del punto de arribo de todo el proceso queda abierta. Para los avances en ciencia esto significa que, respecto del resultado final del proceso de autodesenvolvimiento de los conceptos, sólo se puede prever que éstos serán más configurados o que tendrán una forma más enriquecida y concreta. Por eso reconocer la libertad de la ciencia implica estar dispuesto a asumir coherentemente todas las consecuencias: estar dispuesto, por ejemplo, a todo tipo de sorpresas y, si bien "no todo lo que se puede hacer es lo que se debe hacer", también es verdad que es la ciencia misma a quien le cabe la libertad y responsabilidad de establecer sus propios límites. El verdadero crecimiento científico presupone siempre su libre ejercicio y la comunicación con las otras disciplinas $\mathrm{u}$ otros sujetos epistemológicos sin pretender predeterminar los resultados.

Pero antes de poner punto final a este trabajo considero muy importante, en este punto, hacer referencia a la interpretación de un hegeliano del calibre de Cime-Lima, para quien la lógica dialéctica de Hegel requiere, para dar lugar a esta apertura para libertad, del agregado de un operador modal deóntico que permitiría interpretar la antítesis no como una contradicción de la tesis, sino que tendría, en realidad, con respecto a ella, una relación de contrariedad; por lo que no anularía necesariamente a la primera afirmación; sino que abrińa otras posibilidades: un espacio de contingencia, en el mundo de los fenómenos y un espacio hacia lo eventual, para la libertad del hombre ${ }^{9}$. Sin esta corrección, dicha apertura no sería posible.

Op. cit, p. 815 y Dialética para principiantes, Edipúcrs, Porto Alegre, 1997, 2a. Ed., III, 2. 